\title{
Vitamin B12 deficiency leading to Depression
}

\section{Dr Sneha Arya ${ }^{1}$, Dr Sanjiv S. Kale ${ }^{2}$, Dr Aparna Ramakrishnan ${ }^{3}$}

${ }^{1} 2^{\text {nd }}$ year post graduate student, Dr. DY Patil University, Navi Mumbai, Navi Mumbai. Contact: 9619365764 Email: aryasneha@gmail.com, Contact: 9619365764

${ }^{2}$ Head of Department, Psychiatry; Dr. DY Patil University, Navi Mumbai, Navi Mumbai Email: sanjiVkale58@gmail.com, Contact: 9820547252

${ }^{3}$ Associate Professor, Department of Psychiatry, Dr. DY Patil University, Navi Mumbai, Navi Mumbai Email:aparna.214r@gmail.com, Contact: 9819435861

Abstract
Vitamin B12, also known as cobalamin is a water soluble vitamin, playing a key role in neuronal
functions of the brain, nervous system and cellular metabolism especially DNA synthesis formation.
Deficits in vitamin B12 have negative consequences on the developing brain during infancy and are
associated with a greater risk of depression during adulthood.
Keywords: Vitamin B12; childhood depression.

\section{Introduction}

Vitamin B12 is important for bodily functions. Its deficiency may result in generalised fatigability and decreased energy levels along with hematological and neurological symptoms.

Other symptoms of Vitamin B 12 deficiency include:

1. Mood changes.

2. Irritability.

3. Depression.

4. Psychosis.

5. Lack of appetite.

6. Breathlessness.

7. Confusion.

8. Memory and concentration problems.

9. Sleep disorders.

\section{Case History}

We report a case of a patient diagnosed with Major depressive disorder who showed inadequate response to conventional anti- depressant therapy and hence was investigated; found to have Vitamin B12 deficiency and treated successfully for the same. Significant improvement was seen in depressive symptoms also.

\section{Case}

A 12 year old boy born out of a non consanguineous marriage, full term normal, hospital delivered, with normal developmental milestones from a Hindu orthodox family was brought by parents to psychiatry OPD of a tertiary care hospital with chief complaints of: school refusal, sadness of mood, fatigue, lack of interest, sleep disturbance, irritability, crying spells since 4 months. 


\section{On examination}

Neurological examination revealed no deficits. The child did not show any evidence of Intellectual Disability.

\section{Treatment History}

Patient's first consultation was with a paediatrician. There was no clinical improvement over a period of 3 to 4 months following which patient was referred to a psychiatrist.

Patient was diagnosed as a case of major depressive disorder and was started on Escitalopram 5mg; gradually increased to $20 \mathrm{mg}$ over a period of 6 to 8 weeks. No significant improvement was perceived.

\section{Investigations}

1. Complete blood count ( $\mathrm{CBC})$ : HB: 12.7 gm/dl ; RBC : 3.35 mill/c.mm ; MCV : $109.3 \mathrm{fl} ; \mathrm{MCH}: 37.9 \mathrm{pg}$;

MCHC: 34.7 g/dl; PlateletsS- 286 x 1000 /c.mm

2. Serum vitamin B12: $126 \mathrm{pg} / \mathrm{ml}$.

Rest of the investigations like liver function test, renal function test, blood sugar levels, thyroid function test were found to be within normal limits.

\section{Course of Therapy}

Patient received a series of Vitamin B12 oral supplements over a period of 15 to 20 days till serum Vitamin B12 levels were restored.

The patient showed significant clinical improvement in terms of depressive symptoms.

Treatment response was quite dramatic in this case.

\section{Discussion}

There are several theories concerning potential associations between depression and low levels of Vitamin B12 and Folate .Viatmin B12 and Folate are connected with the synthesis of monoamines and are involved in single carbon transfer methylation reactions connected with the production of monoamine neurotransmitters. Vitamin B12 deficiency may also result in accumulation of homocysteine which has been suggested to lead to excito-toxic reactions and may enhance depression. ${ }^{1}$ Bottiglieri et all found that some depressed patient have lower levels of Vitamin B12 which might reflect lower intake of vitamin's from food due to lack of appetite which is a symptom in depression. ${ }^{2}$

Other risk factors for Vitamin B12 deficiency include:

1. Vegetarian diet.

2. Any intestinal infection or intestinal surgery leading to malabsorption of vitamins.

3. Lack of intrinsic factor.

4. Autoimmune disorder: Diabetes, Thyroid disorder, pernicious anaemia.

In this case, culture bound dietary practices like vegetarianism may have led to Vitamin B12 deficiency. Adequate treatment with antidepressants only did not result in significant clinical improvement in the patient without Vitamin B12 supplementation.

This was a finding similar to other studies. Mischoulan showed that the treatment with SSRI's ( selective serotonin reuptake inhibitor) alone was less effective in patient with Vitamin B12 deficiency. ${ }^{3}$ Hintika J, showed that patient with high Vitamin B12 levels respond better than those with low Vitamin B12 levels. ${ }^{4}$

\section{Conclusion}

In conclusion, Vitamin B12 levels should be evaluated in depressed patients especially if they show inadequate response to adequate therapeutic regimen. Increase in low Vitamin B12 levels may result in dramatic improvement in depressive symptoms which may previously be seen to be difficult to treat. Also psycho-education of patient and family members about importance of nutritional dietary practices for optimum physical and mental health is essential.

\section{References}

1. Herrmann W, Lorenzl S, Obeid R. review of the role of hyperhomocystenemia and B- vitamin deficiency in neurological and psychiatric disorders: current evidence and 
preliminary recommendations. Fortschr Neurol Psychiatry 2009; 75(9) 515-27.

2. Bottliglieri T, Laundy M, Crellin R, et all. Homocysteine, folate, methylation and monoamine metabolism in depression. $\mathrm{J}$ NeurolNeurosurg Psychiatry 2000; 157 (5) : 715-21.

3. Mischoulan D, Burger JK, Spillmann MK, Worthington JJ, Fava M, Alpert JE. Anemia and macrocytosis in the prediction of serum folate and Vitamin B12 status and treatment outcome in major depression. J Psychosom Res 2000; 49 (3) : 183-7.

4. Hintikka J, Tolmunen $\mathrm{T}$, Tanskanen A, Viinamaki H. High vitamin B12 level and good treatment outcome may be associated in major depressive disorder. BMC Psychiatry 2003; 3(1) : 17-8. 